\title{
Incorporating truncating variants in PALB2, CHEK2, and ATM into the BOADICEA breast cancer risk model
}

\author{
Andrew J. Lee, MSc, CASM'1 ${ }^{1}$ Alex P. Cunningham, MSc, PhD11, Marc Tischkowitz, MD, PhD², \\ Jacques Simard, PhD ${ }^{3,4}$, Paul D. Pharoah, $\mathrm{PhD}^{1,5}$, Douglas F. Easton, $\mathrm{PhD}^{1,5}$ and Antonis C. Antoniou, $\mathrm{PhD}^{1}$
}

Purpose: The proliferation of gene panel testing precipitates the need for a breast cancer (BC) risk model that incorporates the effects of mutations in several genes and family history $(\mathrm{FH})$. We extended the BOADICEA model to incorporate the effects of truncating variants in PALB2, CHEK2, and ATM.

Methods: The $B C$ incidence was modeled via the explicit effects of truncating variants in $B R C A 1 / 2, P A L B 2, C H E K 2$, and $A T M$ and other unobserved genetic effects using segregation analysis methods.

Results: The predicted average BC risk by age 80 for an ATM mutation carrier is $28 \%, 30 \%$ for $C H E K 2,50 \%$ for $P A L B 2$, and $74 \%$ for $B R C A 1$ and $B R C A 2$. However, the $\mathrm{BC}$ risks are predicted to increase with $\mathrm{FH}$ burden. In families with mutations, predicted risks for mutation-negative members depend on both $\mathrm{FH}$ and the specific mutation. The reduction in $\mathrm{BC}$ risk after negative predictive testing is greatest when a BRCA1 mutation is identified in the family, but for women whose relatives carry a CHEK2 or ATM mutation, the risks decrease slightly.

Conclusions: The model may be a valuable tool for counseling women who have undergone gene panel testing for providing consistent risks and harmonizing their clinical management. A Web application can be used to obtain BC risks in clinical practice (http://ccge. medschl.cam.ac.uk/boadicea/).

Genet Med advance online publication 14 April 2016

Key Words: ATM; breast cancer risk prediction; CHEK2; gene panel; $P A L B 2$

\section{INTRODUCTION}

Breast cancer (BC) exhibits strong familial aggregation, such that the risk of the disease increases with increasing number of affected relatives. First-degree relatives of women diagnosed with $\mathrm{BC}$ are at approximately twice the risk of developing $\mathrm{BC}$ than women in the general population. ${ }^{1}$ Many BC-susceptibility variants have been identified to date. Approximately $15-20 \%$ of this excess familial risk is explained by rare, high-penetrance mutations in $B R C A 1$ and $B R C A 2{ }^{2,3}$ Other rare, intermediate risk variants (e.g., PALB2, CHEK2, and ATM) are estimated to account for $\sim 5 \%$ of the BC familial aggregation, ${ }^{4-6}$ and the common, lowrisk alleles account for a further $14 \%$ of familial risk. ${ }^{7,8}$

To provide comprehensive genetic counseling for $\mathrm{BC}$, it is important to have risk-prediction models that take into account the effects of all the known susceptibility variants and also account for the residual familial aggregation. Some existing genetic risk-prediction algorithms incorporate the effects of $B R C A 1$ and BRCA2 mutations, including BRCAPRO, ${ }^{9} \mathrm{IBIS},{ }^{10}$ and BOADICEA., ${ }^{3,11}$ BOADICEA accounts for the residual familial aggregation of $\mathrm{BC}$ in terms of a polygenic component that models the multiplicative effects of a large number of variants, with each making a small contribution to the familial risk. ${ }^{3}$
Next-generation sequencing technologies that enable simultaneous sequencing of multiple genes through gene panels ${ }^{12,13}$ have now entered clinical practice. However, the clinical utility of results from such genetic testing remains limited because none of the currently available risk-prediction models incorporate the simultaneous effects of rare intermediate-risk variants and other risk factors, particularly explicit family history. As a result, providing risk estimates for women who carry these mutations, and their relatives, is problematic. ${ }^{6}$

In this article, we describe an extension to the BOADICEA model to incorporate the effects of intermediate risk variants for BC, specifically loss-of-function mutations in the three genes for which the evidence for association is clearest and the risk estimates are most precise: PALB2, CHEK2, and ATM. The resulting model allows for consistent $\mathrm{BC}$ risk prediction in unaffected women on the basis of their genetic testing and their family history.

\section{BC incidence in BOADICEA \\ MATERIALS AND METHODS \\ We build on the existing BOADICEA model. ${ }^{2,3,11}$ Briefly, in this model the $\mathrm{BC}$ incidence, $\lambda_{i}(t)$, for individual $i$ at age $t$ is assumed}

${ }^{1}$ Department of Public Health and Primary Care, Centre for Cancer Genetic Epidemiology, The University of Cambridge, Strangeways Research Laboratory, Cambridge, UK; ${ }^{2}$ Department of Medical Genetics and National Institute for Health Research, Cambridge Biomedical Research Centre, The University of Cambridge, Cambridge, UK;

${ }^{3}$ Canada Research Chair in Oncogenetics, Faculty of Medicine, Université Laval, Quebec City, Quebec, Canada; ${ }^{4}$ Genomics Center, Centre Hospitalier Universitaire de Québec Research Center, Quebec City, Quebec, Canada; ${ }^{5}$ Department of Oncology, Centre for Cancer Genetic Epidemiology, The University of Cambridge, Strangeways Research Laboratory, Cambridge, UK. Correspondence: Antonis C. Antoniou (antonis@srl.cam.ac.uk)

Submitted 29 October 2015; accepted 1 February 2016; advance online publication 14 April 2016. doi:10.1038/gim.2016.31 
to be birth cohort-specific and to depend on the underlying $B R C A 1$ and $B R C A 2$ genotypes and the polygenotype through a model of the form:

$$
\lambda_{i}(t)=\lambda_{0}(t) \exp \left(\beta_{1}(t) G_{1 i}+\beta_{2}(t)\left(1-G_{1 i}\right) G_{2 i}+P_{i}(t)\right),
$$

where $\lambda_{0}(t)$ is the baseline incidence for the cohort, $G_{1 i}$ is an indicator variable taking a value of 1 if a BRCA1 mutation is present and 0 otherwise, and, similarly, $G_{2 i}$ is an indicator for $B R C A 2 . \beta_{1}(t)$ and $\beta_{2}(t)$ represent the age-specific log-relative risks (RRs) associated with BRCA1 and BRCA2 mutations, respectively, relative to the baseline incidence (applicable to a nonmutation carrier with a zero polygenic component) and where $P_{i}(t)$ is the polygenic effect, which is assumed to be normally distributed with mean 0 and variance $\sigma_{P}^{2}(t)$.

The effects of mutations in BRCA1 and BRCA2 are modeled through a single-locus "major gene" with three alleles (BRCA1, $B R C A 2$, and wild-type). The BRCA1 and BRCA2 alleles are assumed to be dominantly inherited. ${ }^{14}$ As a further simplification, carriers of both the BRCA1 and BRCA2 alleles are assumed to be susceptible to $B R C A 1$ risks. These simplifications reduce the number of possible "major" genotypes from nine to three: a $B R C A 1$ mutation carrier, a BRCA 2 mutation carrier, and a nonmutation carrier.

The BOADICEA genetic model uses the Elston-Stewart peeling algorithm to compute the pedigree likelihood. ${ }^{15,16}$ As a result, the number of computations increases exponentially with the number of possible genotypes. To maintain computational efficiency, we incorporated the effects of risk-conferring variants in PALB2, CHEK2, and ATM into the model by introducing an additional allele for each gene (representing a mutation in that gene) to the major gene locus, resulting in a locus with six alleles. In comparison with a model that has a single locus for each gene, this approximation is justified by the low

Table 1 Mutation frequency and relative risk for loss-offunction variants in PALB2, CHEK2, and ATM

\begin{tabular}{lccc} 
& PALB2 & CHEK2 & ATM \\
\hline Allele frequency & $0.057 \%$ & $0.26 \%$ & $0.19 \%$ \\
Age & \multicolumn{1}{c}{ Relative risk (95\% Cl) } \\
\hline $20-24$ & $9.01(5.70-14.16)$ & & \\
$25-29$ & $8.97(5.68-14.08)$ & & \\
$30-34$ & $8.85(5.63-13.78)$ & & \\
$35-39$ & $8.54(5.51-13.08)$ & & \\
$40-44$ & $8.03(5.29-11.95)$ & & \\
$45-49$ & $7.31(4.98-10.55)$ & $3.0(2.6-3.5)$ & $2.8 .7)$ \\
$50-54$ & $6.55(4.60-9.18)$ & & \\
$55-59$ & $5.92(4.27-8.10)$ & & \\
$60-64$ & $5.45(4.00-7.33)$ & & \\
$65-69$ & $5.10(3.80-6.76)$ & & \\
$70-74$ & $4.82(3.63-6.33)$ & & \\
$75-79$ & $4.56(3.48-5.95)$ & & \\
\hline
\end{tabular}

The relative risk values for $P A L B 2$ are taken from ref. 20 . The mutation frequency for PALB2 is taken from a private communication from Easton and Pharaoh based on data from unaffected individuals from the United Kingdom. Relative risk values for CHEK2 and ATM are from ref. 6 and are not age specific. The allele frequency

for CHEK2 is from ref. 28, and the allele frequency for ATM from ref. 29. allele mutation frequencies for all genes (Table 1), because the probability of carrying more than one mutation is $l$ low $^{17}$ relative to the probability of carrying one or no mutation. Currently, few published data describe the cancer risks for carrying more than one mutation. ${ }^{18}$ Here, we assumed that the risks follow a dominant model, with the order of precedence being BRCA1, BRCA2, PALB2, CHEK2, ATM, and wild-type. Under this model, in the presence of a mutation in one gene, no additional risk is conferred by a second mutation in another gene lower in the dominance chain.

\section{RRs for female BC}

We extended the model for the $\mathrm{BC}$ incidence to incorporate the effects of variants in PALB2, CHEK2, and ATM, such that:

$$
\lambda_{i}(t)=\lambda_{0}(t) \exp \left(\sum_{\alpha=1}^{5} \beta_{\alpha}(t) \prod_{\gamma=1}^{\alpha-1}\left(1-G_{\gamma i}(t)\right) G_{\alpha i}(t)+P_{R i}(t)\right),
$$

where $\lambda_{0}, \beta_{1}(t), G_{1 i}, \beta_{2}(t)$, and $G_{2 i}$ are as described in equation (1), and $G_{3 i}, G_{4 i}$, and $G_{5 i}$, are indicator variables taking values of 1 if a mutation is present and 0 otherwise for PALB2, CHEK2, and ATM, respectively. $\beta_{3}(t), \beta_{4}(t)$, and $\beta_{5}(t)$ represent the age-specific log-RRs associated with PALB2, CHEK2, and ATM mutations, respectively, relative to the baseline incidence (applicable to a nonmutation carrier with a zero polygenic component). $P_{R i}(t)$ is the residual polygenic component, with mean 0 and variance $\sigma_{R}^{2}(t)$ explained in equation (3), below.

To implement the model in equation (2), we assumed the mutation frequencies and RRs summarized in Table 1. The RR estimates are relative to the population incidences and are therefore over all polygenic effects. Multiplying these RRs by the cohort and age-specific incidences yields the average incidences in carriers of PALB2, CHEK2, and ATM mutations over all polygenic effects. To obtain $\beta_{3}(t), \beta_{4}(t)$, and $\beta_{5}(t)$, we constrained the overall incidences (using as weights the major genotype and polygenic frequencies) to agree with the population $\mathrm{BC}$ incidence for each birth cohort separately. This process is detailed elsewhere. ${ }^{14}$

To ensure that the familial risks predicted by this extended model remain consistent with the previous model, we adjusted the variance of the polygenic component to account for the fact that the contributions of PALB2, CHEK2, and ATM to the genetic variance are now explicitly accounted for in the major gene, following the process described elsewhere. ${ }^{19}$ Briefly, the total polygenic variance $\left(\sigma_{P}^{2}\right)$ was decomposed into the sum of the known variance $\left(\sigma_{K}^{2}\right)$, due to the three variants, and residual variance $\left(\sigma_{R}^{2}\right)$,

$$
\sigma_{P}^{2}=\sigma_{K}^{2}+\sigma_{R}^{2},
$$

The known variance, $\sigma_{K}^{2}$, can be calculated from the RRs and mutation frequencies of the variants. ${ }^{19}$ This assumes the effects of each variant and the residual polygene are multiplicative, which agrees with recent findings for PALB2. ${ }^{20}$ This model is also consistent with the higher RR for CHEK2 1100delC for BC based on familial cases, ${ }^{21,22}$ the higher RR for bilateral $\mathrm{BC},{ }^{23}$ and the increased risk in relatives of $\mathrm{BC}$ patients who are CHEK2 
carriers. ${ }^{24} \mathrm{~A}$ higher RR for familial BC for ATM carriers has also been found, although data are more limited. ${ }^{25}$

\section{PALB2 characteristics}

Because of the small number of case-control studies for PALB2 or $A T M$, we used alternative family-based data. Age-dependent RRs of female BC for carriers of loss-of-function variants in PALB2 were taken from a large collaborative family-based study ${ }^{20}$ With the exception of specific Nordic founder mutations, data on mutation frequencies in the general population are sparse. We assumed a mutation allele frequency of $0.057 \%$ based on data from targeted sequencing of $8,705 \mathrm{UK}$ controls (unpublished data). This is close to the average estimate across published estimates. ${ }^{26}$

\section{CHEK2 characteristics}

Most existing data pertain to the CHEK2 1100delC variant, which is the most common truncating variant in northern European populations. ${ }^{27}$ CHEK2 1100delC has been evaluated in many case-control studies. ${ }^{22,28}$ As a result, we based the CHEK2 estimates on the CHEK2 1100delC carrier estimates from a meta-analysis. ${ }^{28}$ We assumed that the allele frequency of the $1100 \mathrm{delC}$ mutations was $0.26 \%$, which is the combined frequency across unselected population controls of European ancestry. ${ }^{28}$ There is some evidence that the RRs for $\mathrm{BC}$ in CHEK2 1100delC carriers decline with age. ${ }^{22}$ However, because age-specific estimates are currently imprecise, we used a single $\mathrm{RR}$ across all ages.

\section{ATM characteristics}

We obtained estimates for truncating mutations in ATM from a combined analysis of three estimates from cohort studies of relatives of ataxia-telangiectasia (A-T) patients (Table 1). ${ }^{6}$ The great majority of A-T patients carry two truncating ATM mutations, and relatives of A-T patients are therefore known to have a high probability of being carriers of an ATM mutation. We assumed that the allele frequency of truncating variants in ATM was $0.19 \%$, based on data from UK controls. ${ }^{25}$ As for CHEK2, there is some evidence of a decline in RR with age, ${ }^{29}$ but, lacking precise estimates, we used a single estimate across all ages.

\section{RRs for other cancers}

In addition to the risks of female BC, BOADICEA takes into account the associations of BRCA1 and BRCA2 mutations with the risks of male breast, ovarian, pancreatic, and prostate cancers. ${ }^{3}$ Several studies have investigated associations of the truncating variants in PALB2, ATM, and CHEK2 1100delC with the risks of these cancers (and others). ${ }^{20,29-31}$ However, none of the

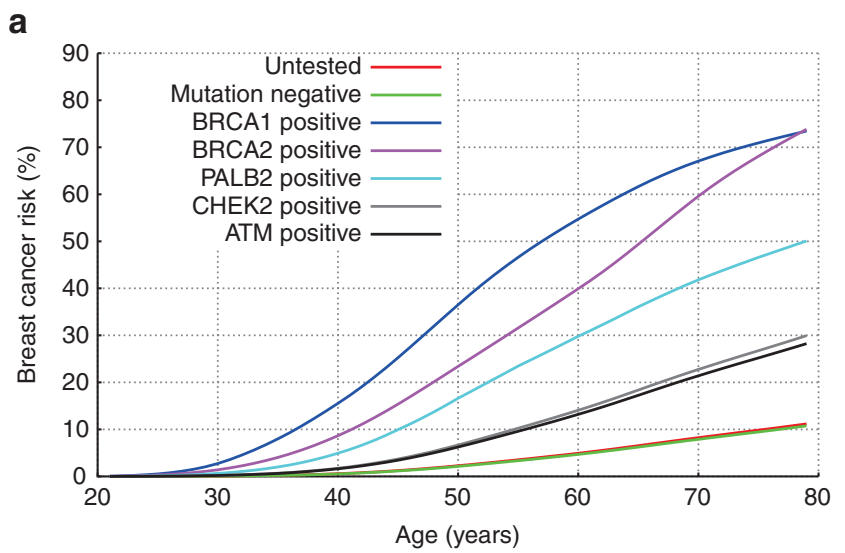

b
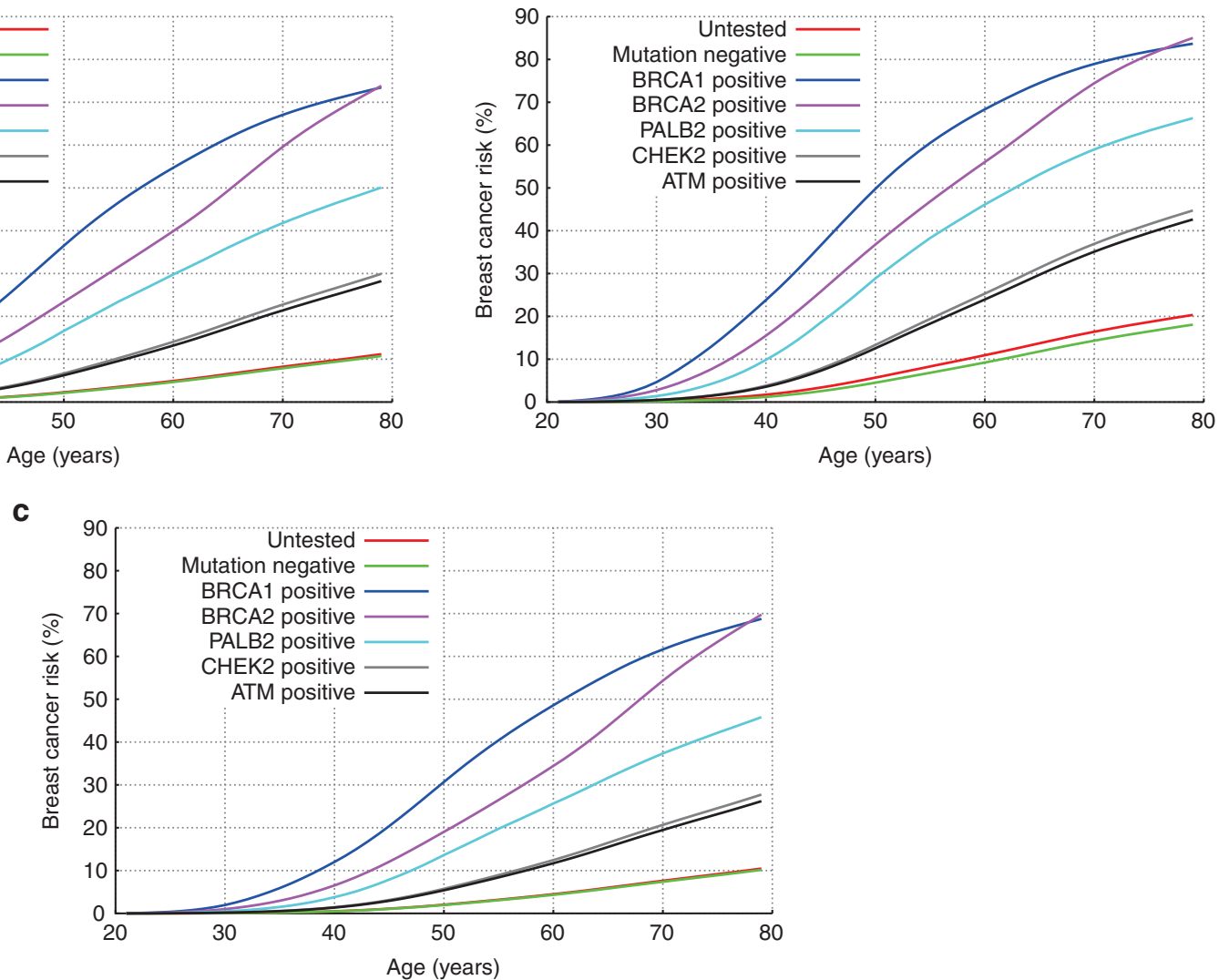

Figure 1 BOADICEA breast cancer (BC) risk by mutation status and family history. BOADICEA risk by mutation status for a female in the United Kingdom 20 years of age and born in 1975 with (a) unknown family history (i.e., for the average female in the population), (b) her mother affected at age 40 years, (c) her mother and sister unaffected at ages 70 years and 50 years, respectively. No testing was assumed in other family members in all cases. 
studies provided convincing evidence of association for any of these cancers, and accurate penetrance estimates are currently lacking for those cancers that may have associations. Therefore, for the purpose of the current implementation, we assumed that these mutations are not associated with risks of other cancers.

\section{Incorporating breast tumor pathology characteristics}

Previous studies ${ }^{2,11}$ have described the incorporation into BOADICEA of differences in tumor pathology subtypes between BRCA1, BRCA2, and noncarrier BCs. Specifically, BOADICEA includes information on tumor estrogen receptor (ER) status, triple negative (estrogen-, progesterone-, and HER2-negative) (TN) status, and the expression of basal cytokeratin markers CK5/6 and CK14.

BCs in CHEK2 1100delC mutation carriers have been found to be ER-positive in a greater proportion compared with tumors in non-CHEK2 mutation carriers ( 88 vs. $78 \%$ in the general population). ${ }^{32}$ Reliable data pertaining to the $\mathrm{TN}$ and basal cytokeratin receptor status are not currently available. Therefore, in the current implementation, we only incorporated differences by BC ER status for CHEK2 1100delC carriers. Age-specific distributions were not available.

Published data on the prevalence of tumor subtypes in PALB2-associated BCs are sparse; although some differences from the general population have been reported, these are based on small numbers. ${ }^{20,33}$ Currently, there are no available data pertaining to tumor pathology subtype distributions for carriers of ATM truncating mutations. We therefore assumed that tumor subtype distributions for PALB2 and ATM mutation carriers are the same as those for the general population.

\section{Mutation screening sensitivity}

We have introduced separate mutation test screening sensitivities for PALB2, CHEK2, and ATM to allow for the fact that some risk-conferring variants in these genes may be missed by current methods. In the BOADICEA Web Application (BWA), we assumed default values of $90 \%$ for PALB2 and ATM truncating variants and of $100 \%$ for the CHEK2 1100delC variant. However, these values can be customized by users. The specificity of mutation testing was assumed to be $100 \%$.

\section{RESULTS}

Figure 1a and Supplementary Figure S1 shows the implied average cumulative $\mathrm{BC}$ risks predicted by BOADICEA by mutation status on the basis of the assumed RRs for an unaffected female 20 years of age. The predicted average breast risk by age 80 years was $28.2 \%$ for an ATM mutation carrier, $29.9 \%$ for CHEK2, $50.1 \%$ for PALB2, 73.5\% for BRCA1, and 73.8\% for BRCA2.

Compiled on the basis of the assumed mutation frequencies and RRs and modeling assumptions, the known polygenic variances $\left(\sigma_{K}^{2}(t)\right)$ due to the effects of PALB2, CHEK2, and ATM are shown in Table 2. The age dependence of the variances due to PALB2, CHEK2, and ATM is a consequence of the fact that RRs vary with age (particularly for PALB2) and the age dependence of the frequency of mutation carriers among the unaffected
Table 2 The variance explained by PALB2, CHEK2, and ATM and the percentage of the overall polygenic variance explained by all three combined

\begin{tabular}{lcccc} 
Age & $\begin{array}{c}\text { PALB2 } \\
\text { variance }\end{array}$ & $\begin{array}{c}\text { CHEK2 } \\
\text { variance }\end{array}$ & $\begin{array}{c}\text { ATM } \\
\text { variance }\end{array}$ & $\begin{array}{c}\text { Total polygenic } \\
\text { variance (\%) }\end{array}$ \\
\hline 25 & 0.0691 & 0.0201 & 0.0121 & 3.04 \\
30 & 0.0668 & 0.0201 & 0.0121 & 3.26 \\
35 & 0.0611 & 0.0201 & 0.0121 & 3.40 \\
40 & 0.0519 & 0.0199 & 0.012 & 3.43 \\
\hline 45 & 0.0403 & 0.0197 & 0.0119 & 3.35 \\
\hline 50 & 0.0294 & 0.0193 & 0.0116 & 3.26 \\
\hline 55 & 0.0216 & 0.0188 & 0.0114 & 3.34 \\
60 & 0.0165 & 0.0182 & 0.0111 & 3.66 \\
65 & 0.013 & 0.0176 & 0.0108 & 4.34 \\
70 & 0.0105 & 0.017 & 0.0104 & 5.77 \\
\hline 75 & 0.0085 & 0.0164 & 0.0101 & 9.77 \\
\hline
\end{tabular}

population, which decreases with age (elimination effect). The proportion of polygenic variance accounted for by these genes varied from $3.0 \%$ at age 25 years to $9.8 \%$ at age 75 years.

\section{Mutation carrier probabilities}

Figure 2 shows the mutation carrier probabilities predicted by BOADICEA for a female with unknown family history as a function of her age at cancer diagnosis, and for a 30-yearold female diagnosed with BC (whose mother has had BC) as a function of her mother's age at diagnosis (also shown in Supplementary Tables S1 and S2 online). The mutation carrier probabilities for ATM and CHEK2 did not show a marked change with age at diagnosis (reflecting the assumption of a constant RR), but the mutation carrier probabilities for PALB2 decreased with age, although less markedly than for BRCA1/2. The mutation carrier probabilities were higher for women with a family history, but the effect was more marked for BRCA1/2 and PALB2 than for CHEK2 or ATM.

\section{Predicted cancer risks for mutation carriers are family history-specific}

In our model, the residual polygenic component was assumed to act multiplicatively with PALB2, CHEK2, and ATM mutations on $\mathrm{BC}$ risk. As a result, the risks for mutation carriers will vary by family history. Figure 1 shows the predicted cumulative $\mathrm{BC}$ risk for a 20 -year-old woman by her mutation status. In (a), the woman is assumed to have unknown family history. In (b), the woman is assumed to have a mother affected with $\mathrm{BC}$ at age 40. In (c), the woman has a mother and sister who are cancer-free at ages 70 years and 50 years, respectively. These clearly show that predicted $\mathrm{BC}$ risks increased with an increasing number of affected relatives and depend on the phenotypes of unaffected family members. For example, although the average $\mathrm{BC}$ risks by age 80 years for CHEK2 and ATM mutation carriers were lower than $30 \%$ (a common criterion for "high" risk, e.g., the NICE guideline ${ }^{34}$ ), the $\mathrm{BC}$ risk exceeded this threshold when a mutation carrier had a family history of $\mathrm{BC}$ (e.g., $42.6 \%$ for ATM and $44.7 \%$ for CHEK2 with an affected mother). 
a

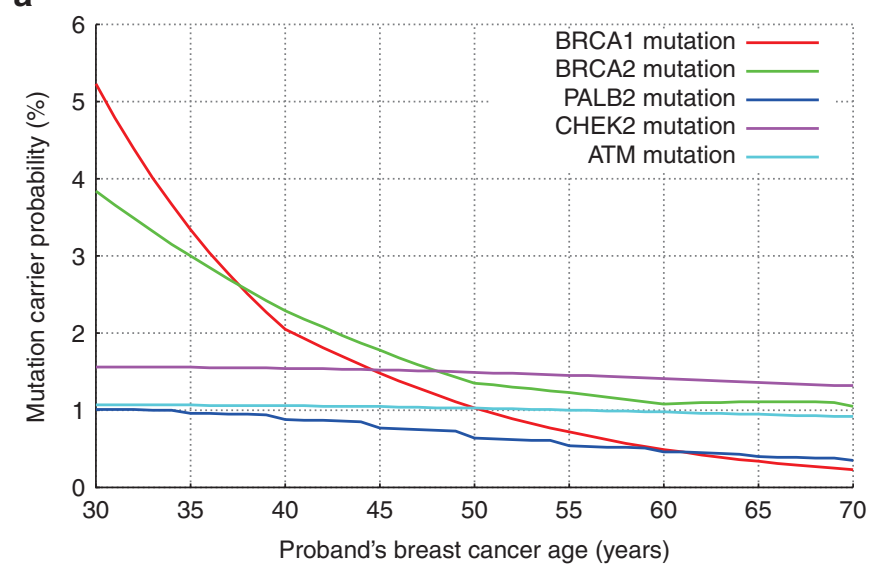

b

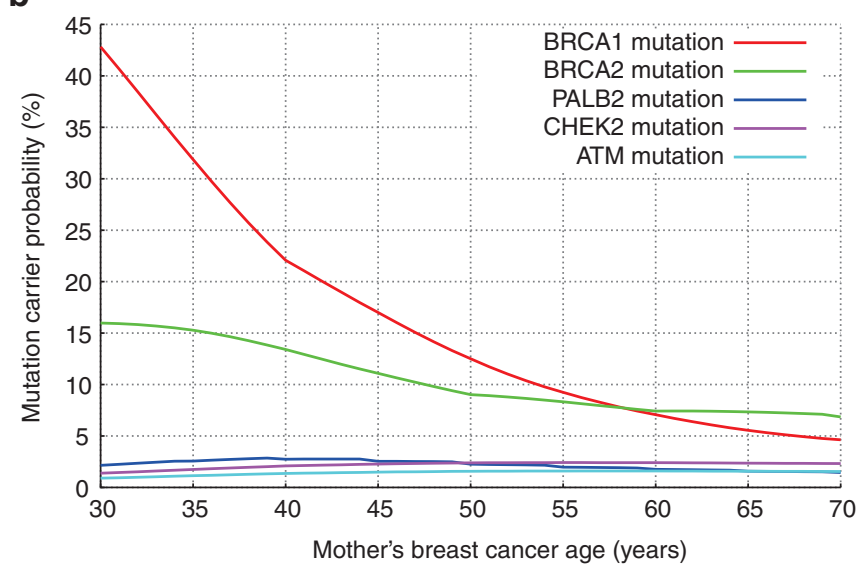

Figure 2 BOADICEA mutation carrier probabilities. BOADICEA mutation carrier probabilities for a female in the United Kingdom born in 1975 (a) with unknown family history as a function of her breast cancer (BC) diagnosis age and (b) who was diagnosed with $B C$ at age 30 years. Her mother was diagnosed with $B C$ as a function of her mother's age at diagnosis.

Comparing Figure 1a and 1c, we see that the risk for a woman with no history of $\mathrm{BC}$ is lower than the average $\mathrm{BC}$ risk.

\section{The effect of negative predictive testing}

The extended BOADICEA model can also be used to predict risks in families in which mutations are identified but other family members test negative. This is demonstrated for a number of family history scenarios in Figure 3, which depend on the mutation status of the proband and her mother. The predicted risks for mutation-negative family members depend on both the family history and the specific mutation identified. Thus, for families with a history of BC, namely, Figure 3c, e, and $\mathrm{g}$, the reduction in $\mathrm{BC}$ risk after negative predictive testing is greatest when a BRCA1 mutation was identified in the family, with the risks being close to (although still somewhat greater than) the population risk. This effect was most noticeable for women with a strong family history. The reduction for women whose mother carried a BRCA2 or PALB2 mutation is less marked, whereas for women whose mother carried a CHEK2 or an ATM mutation, the risks decreased only slightly with a negative predictive test, even with a strong family history. For a woman with no history (Figure 3a), her risk based on the family history alone (i.e., in an untested family) was slightly lower than that of the population risk. After negative predictive testing, her predicted risk decreased further. The biggest decrease was observed when a BRCA1 mutation was identified in the mother.

\section{Updates to the BWA}

We have now updated the BWA (http://ccge.medschl.cam. ac.uk/boadicea/) to accommodate these extensions to the BOADICEA model. The BWA enables users to either build a pedigree online or upload pedigrees. When users build an input pedigree online, the program now enables users to specify PALB2, CHEK2, and ATM genetic test results. Similarly, we have extended the BOADICEA import/export format (described in
Appendix A of the BWA v4 user guide: https://pluto.srl.cam. ac.uk/bd4/v4/docs/BWA_v4_user_guide.pdf) so that users can include this information in their files.

\section{DISCUSSION}

Cost-effective sequencing technologies have brought multigene panel testing into mainstream clinical care. ${ }^{6,13,35}$ Although several established BC-susceptibility genes are included in these panels, their clinical utility is limited by the lack of riskprediction models that consider the effects of mutations in these genes and other risk factors, particularly family history. Here, we present an extended BOADICEA model that incorporates the effects of rare protein truncating variants in $P A L B 2$, $C H E K 2$, and ATM. This is the first BC risk model to include the explicit effects of susceptibility genes other than BRCA1 and $B R C A 2$, and it can be used to provide comprehensive risk counseling on the basis of family history and mutation screening of the five genes. The model can also be used to predict risks of developing $\mathrm{BC}$ and the likelihood of carrying truncating mutations in any of the five genes.

The extended BOADICEA model is based on a number of assumptions. To ensure that the model is computationally efficient, we used a single "major" locus with six alleles representing the truncating variants in the five genes and a wild-type allele. In comparison with a model consisting of five separate loci each with two alleles, this should be a reasonable approximation because all the variants are rare. However, it is possible that the effects will be greater in families segregating more than one rare variant. It also represents a substantial reduction in the number of genotypes (36 vs. 1024), and in execution time; we measured execution time and found it was reduced by a factor of 21,000 . These simplifications will become more critical as the number of susceptibility genes included in the model increases. A previous study ${ }^{6}$ identified six other genes for which the association with BC was well established (TP53, PTEN, STK11, CDH1, NF1, and $N B N$ ), and this list is likely to increase with time. 


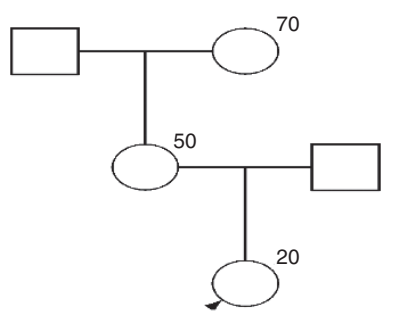

C

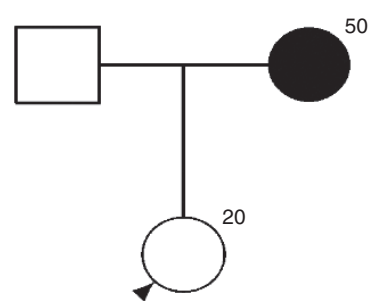

e

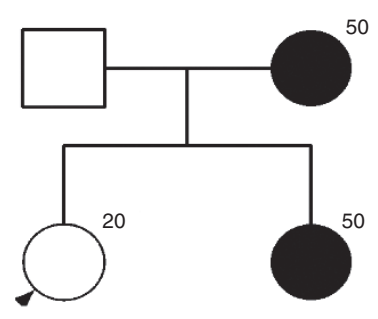

g

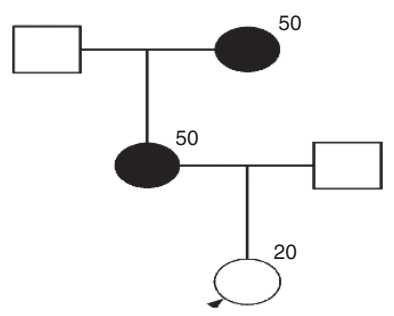

b

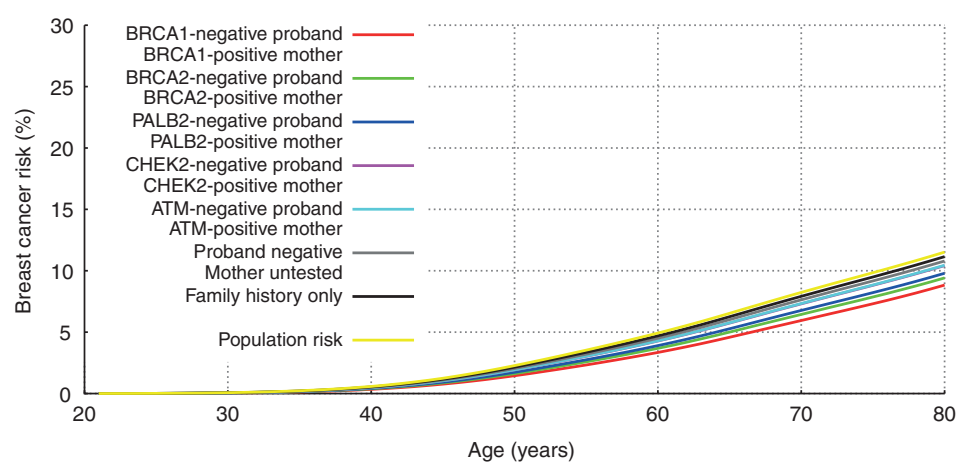

d

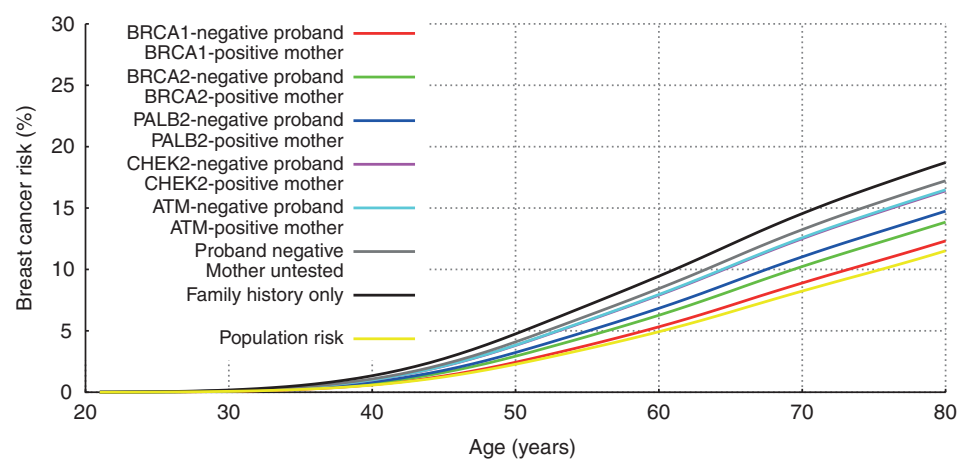

$\mathbf{f}$

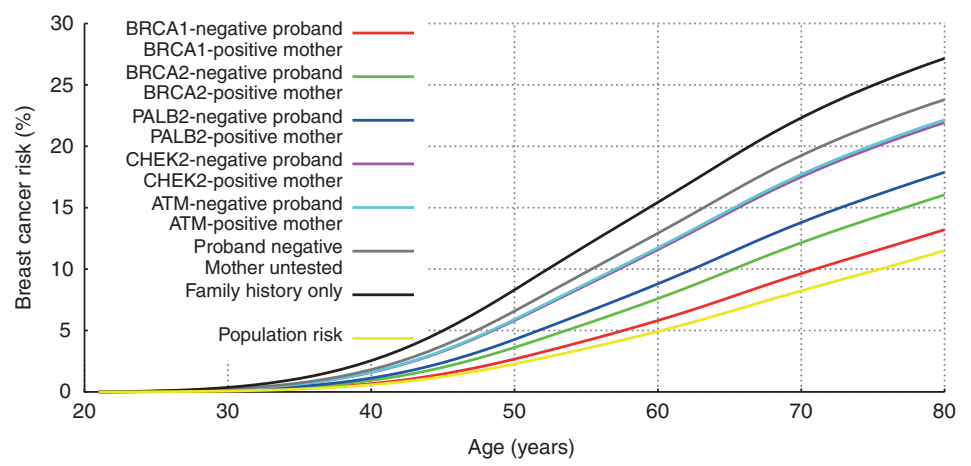

h

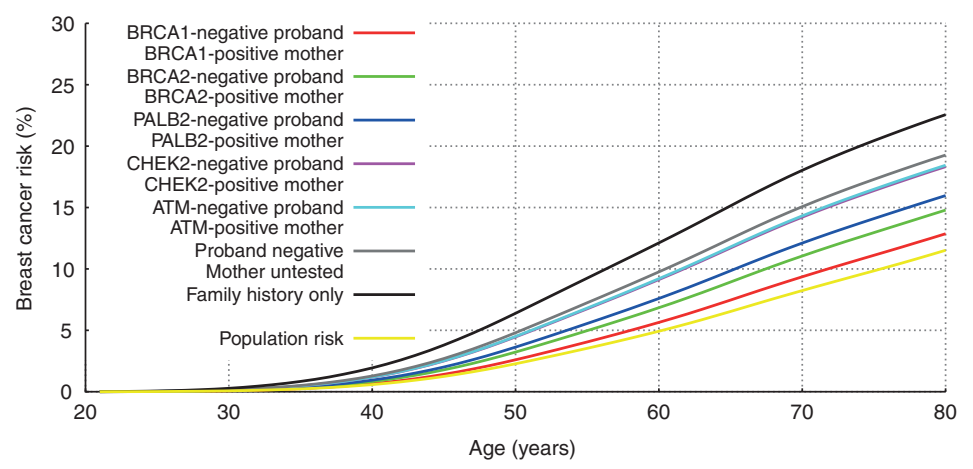

Figure 3 BOADICEA breast cancer (BC) risk for negative testing by family history. The predicted risk of BC by her mother's mutation status and for different family histories for a 20-year-old woman in the United Kingdom born in 1975. The predicted risk is shown for four different family histories. The graphs (b), (d), (f) and (h) correspond to risks for the pedigrees $(\mathbf{a}),(\mathbf{c}),(\mathbf{e})$ and $(\mathbf{g})$, respectively. The figures show the predicted risks for a proband (arrow) in families without any mutation testing in the five genes, i.e., this corresponds to the predicted risk on the basis of family history information alone (black curves). The rest of the curves correspond to the cases where the proband is assumed to be negative for the mutation identified in the family. To enable direct comparisons, the proband is assumed to be 20 years old in all examples. 


\section{ORIGINAL RESEARCH ARTICLE}

Without robust data on risks to carriers of two or more truncating variants (in different genes), we assumed that dual mutation carriers develop BC according to incidences for the higher penetrance gene. Recent evidence suggests that gene-gene interaction between CHEK2, ATM, BRCA1, and BRCA2 may not be multiplicative (indeed, a multiplicative model would be implausible for BRCA1 and BRCA2 because it would predict an extremely high risk at very young ages). ${ }^{18}$ This may reflect the biological relationships between the proteins encoded by the genes. The proteins encoded by all five genes play roles in DNA repair, and loss-of-function mutations in these genes are predicted to impair DNA repair. Our implementation would be consistent with a model in which if the pathway is disrupted by one mutation, then further disruption by a lower penetrance mutation would not increase risk.

Although there is strong evidence that mutations in PALB2, CHEK2, and ATM confer increased risk of BC in females, ${ }^{6}$ there are currently no precise risk estimates for the other cancers considered by BOADICEA (male breast, ovarian, pancreatic, or prostate) or other cancers. However, several studies have provided tentative evidence of associations..$^{20,31}$ Because of the lack of precise cancer risk estimates, we have assumed no association between truncating variants in PALB2, CHEK2, and $A T M$ (i.e., $\mathrm{RR}=1$ ). If there are true associations between the PALB2, CHEK2, and ATM truncating variants and other cancer risks, then we expect that PALB2, CHEK2, and ATM mutation carrier probabilities may potentially be underestimated in families where other cancers occur. However, if accurate risk estimates become available, then they can easily be included in our implementation.

BOADICEA allows cancer tumor characteristics to be taken into account, as we have done previously for $B R C A 1$ and $B R C A 2$ (refs. 2,11,36). The provision of subtype-specific risks can be useful for genetic counseling and may guide chemoprevention. However, data on the additional genes are currently sparse. In this model, we incorporated a higher probability of ER-positive tumors in CHEK2 1100delC carriers relative to noncarriers. ${ }^{32}$ Some studies have suggested differences in the tumor characteristics from PALB2 mutation carriers and noncarriers, but larger studies are required to establish such differences. ${ }^{20,33}$

We considered only the effects of truncating variants in PALB2, ATM, and the CHEK2 1100delC variant, for which robust $\mathrm{BC}$ risk estimates are available. In doing this, we are making the usual simplification that all truncating variants in these genes confer similar risks. Although there is no evidence to contradict this, it may change as further data accumulate. Also, there is evidence that missense variants in CHEK2 and ATM confer elevated BC risks, but that the risks that they confer can differ from the risks associated with truncating variants. For example, the ATM c.7271T $>\mathrm{G}$ missense variant has been reported to confer a higher risk than truncating variants, but the confidence intervals associated with this estimate are currently wide. ${ }^{37}$ It has been suggested that other rare, evolutionarily unlikely missense variants in ATM are also associated with increased BC risks. ${ }^{38}$ Future extensions of BOADICEA can accommodate such differences on the basis of more precise cancer risk estimates. In CHEK2, the missense variant Ile157Thr has been associated with a lower risk than the 1100delC variant. ${ }^{39}$ This variant has been incorporated into a polygenic risk score on the basis of common genetic variants, ${ }^{40}$ which we expect to incorporate into BOADICEA in the future. The model could also be applicable for other truncating variants in CHEK2, under the assumption that they confer risks similar to those of the 1100delC variant. However, the available data are scarce and some modification of the mutation frequencies may be required.

Under the BOADICEA model, women testing negative for known familial mutations (true negatives) and who have family history are predicted to be at higher risk for $\mathrm{BC}$ than the general population. The level of risk depends on both family history and the specific mutation identified. So far, epidemiological studies have reported estimates for "true negatives" only for families with BRCA1 and BRCA2 mutations, ${ }^{41-46}$ but the estimated RRs (compared with the population risks) vary widely. Moreover, all the reported estimates are associated with wide confidence intervals because the studies have been based on small sample sizes. The reported estimates are summarized in Table S3. To provide a direct comparison with the predicted risks by BOADICEA, we have included the implied RRs for the true negative women in Figure 3 relative to the population. These are all in line with the published estimates for true negatives. Therefore, the predictions by BOADICEA are consistent with published data. It is worth noting that if the true RRs for the true negatives in families with $B R C A 1$ and $B R C A 2$ mutations are in line with those predicted by BOADICEA, then very large prospective studies of true negatives would be necessary to demonstrate significant associations.

The current model is a synthetic model based on segregation analyses of families in the United Kingdom together with risk estimates derived from studies of European populations. We have previously implemented procedures for extrapolating the model to populations with different baseline incidence rates based on the assumption that the RRs conferred by the genetic variants in the model are independent of the population. ${ }^{11}$ Thus, the model should be broadly applicable to developed populations of European ancestry, but its applicability to populations with lower incidence rates and populations of non-European ancestry has yet to be evaluated. The implementation also allows the allele frequencies to be adjusted. This may be particularly relevant for CHEK2; in European populations, the founder $1100 \mathrm{delC}$ variant accounts for the majority of carriers of truncating variants and its frequency varies across populations.

The extended BOADICEA model presented here has addressed a major gap in $\mathrm{BC}$ risk prediction by including the effects of truncating variants in PALB2, CHEK2, and ATM that are included in widely used commercial gene panels. The model could be a valuable tool in the counseling process of women who have undergone gene panel testing, because it provides consistent $\mathrm{BC}$ risks and thus harmonizes the clinical 
management of at-risk individuals. Future studies should aim to validate this model in large prospective cohorts with mutation screening information and to evaluate the impact of the risk predictions on decision making.

\section{SUPPLEMENTARY MATERIAL}

Supplementary material is linked to the online version of the paper at http://www.nature.com/gim

\section{ACKNOWLEDGMENTS}

This work was funded by Cancer Research UK Grants C12292/ A11174 and C1287/A10118. A.C.A. is a Cancer Research UK Senior Cancer Research Fellow. This work was supported by the Government of Canada through Genome Canada and the Canadian Institutes of Health Research, and the Ministère de I'Enseignement Supérieur, de la Recherche, de la Science, et de la Technologie du Québec through Génome Québec.

\section{DISCLOSURE}

The authors declare no conflicts of interest.

\section{REFERENCES}

1. Collaborative Group on Hormonal Factors in Breast C. Familial breast cancer: collaborative reanalysis of individual data from 52 epidemiological studies including 58,209 women with breast cancer and 101,986 women without the disease. Lancet 2001;358:1389-1399.

2. Mavaddat N, Rebbeck TR, Lakhani SR, Easton DF, Antoniou AC. Incorporating tumour pathology information into breast cancer risk prediction algorithms. Breast Cancer Res 2010;12:R28.

3. Antoniou AC, Cunningham AP, Peto J, et al. The BOADICEA model of genetic susceptibility to breast and ovarian cancers: updates and extensions. Br J Cancer 2008;98:1457-1466.

4. Apostolou P, Fostira F. Hereditary breast cancer: the era of new susceptibility genes. Biomed Res Int 2013;2013:747318

5. Thompson D, Easton D. The genetic epidemiology of breast cancer genes. J Mammary Gland Biol Neoplasia 2004;9:221-236.

6. Easton DF, Pharoah PD, Antoniou AC, et al. Gene-panel sequencing and the prediction of breast-cancer risk. N Engl J Med 2015;372:2243-2257.

7. Michailidou K, Hall P, Gonzalez-Neira A, et al.; Breast and Ovarian Cancer Susceptibility Collaboration; Hereditary Breast and Ovarian Cancer Research Group Netherlands (HEBON); kConFab Investigators; Australian Ovarian Cancer Study Group; GENICA (Gene Environment Interaction and Breast Cancer in Germany) Network. Large-scale genotyping identifies 41 new loci associated with breast cancer risk. Nat Genet 2013;45:353-61, 361e1.

8. Michailidou K, Beesley J, Lindstrom S, et al.; BOCS; KConFab Investigators; AOCS Group; NBCS; GENICA Network. Genome-wide association analysis of more than 120,000 individuals identifies 15 new susceptibility loci for breast cancer. Nat Genet 2015;47:373-380.

9. Parmigiani G, Berry D, Aguilar O. Determining carrier probabilities for breast cancersusceptibility genes BRCA1 and BRCA2. Am J Hum Genet 1998;62:145-158.

10. Tyrer J, Duffy SW, Cuzick J. A breast cancer prediction model incorporating familial and personal risk factors. Stat Med 2004;23:1111-1130.

11. Lee AJ, Cunningham AP, Kuchenbaecker KB, Mavaddat N, Easton DF, Antoniou AC; Consortium of Investigators of Modifiers of BRCA1/2; Breast Cancer Association Consortium. BOADICEA breast cancer risk prediction model: updates to cancer incidences, tumour pathology and web interface. $\mathrm{Br} J$ Cancer 2014;110:535-545.

12. Robson M. Multigene panel testing: planning the next generation of research studies in clinical cancer genetics. J Clin Oncol 2014;32:1987-1989.

13. Kurian AW, Hare EE, Mills MA, et al. Clinical evaluation of a multiplegene sequencing panel for hereditary cancer risk assessment. J Clin Oncol 2014;32:2001-2009.

14. Antoniou AC, Pharoah PD, McMullan G, Day NE, Ponder BA, Easton D. Evidence for further breast cancer susceptibility genes in addition to BRCA1 and BRCA2 in a population-based study. Genet Epidemio/ 2001;21:1-18.

15. Lange K, Weeks D, Boehnke M. Programs for pedigree analysis: MENDEL, FISHER, and dGENE. Genet Epidemiol 1988;5:471-472.
16. Lange K. Mathematical and Statistical Methods for Genetic Analysis. Springer: New York, 1997.

17. Tung N, Battelli C, Allen B, et al. Frequency of mutations in individuals with breast cancer referred for BRCA1 and BRCA2 testing using next-generation sequencing with a 25-gene panel. Cancer 2015;121:25-33.

18. Turnbull C, Seal S, Renwick A, et al.; Breast Cancer Susceptibility Collaboration (UK), EMBRACE. Gene-gene interactions in breast cancer susceptibility. Hum Mol Genet 2012;21:958-962.

19. Macinnis RJ, Antoniou AC, Eeles RA, et al. A risk prediction algorithm based on family history and common genetic variants: application to prostate cancer with potential clinical impact. Genet Epidemiol 2011;35:549-556.

20. Antoniou AC, Casadei S, Heikkinen T, et al. Breast-cancer risk in families with mutations in PALB2. N Eng/ J Med 2014;371:497-506.

21. Meijers-Heijboer $H$, van den Ouweland A, Klijn J, et al.; CHEK2-Breast Cancer Consortium. Low-penetrance susceptibility to breast cancer due to CHEK2 $\left.{ }^{*}\right) 1100$ delC in noncarriers of BRCA1 or BRCA2 mutations. Nat Genet 2002;31:55-59.

22. Consortium CBCC-C. CHEK2*1100delC and susceptibility to breast cancer: a collaborative analysis involving 10,860 breast cancer cases and 9,065 controls from 10 studies. Am J Hum Genet 2004;74:1175-1182.

23. Fletcher O, Johnson N, Dos Santos Silva I, et al. Family history, genetic testing, and clinical risk prediction: pooled analysis of CHEK2 1100delC in 1,828 bilateral breast cancers and 7,030 controls. Cancer Epidemiol Biomarkers Prev 2009;18:230-234.

24. Johnson N, Fletcher O, Naceur-Lombardelli C, dos Santos Silva I, Ashworth A, Peto J. Interaction between $\mathrm{CHEK} 2 * 1100 \mathrm{del}$ C and other low-penetrance breast-cancer susceptibility genes: a familial study. Lancet 2005;366: 1554-1557.

25. Renwick A, Thompson D, Seal S, et al.; Breast Cancer Susceptibility Collaboration (UK). ATM mutations that cause ataxia-telangiectasia are breast cancer susceptibility alleles. Nat Genet 2006;38:873-875.

26. Southey MC, Teo ZL, Winship I. PALB2 and breast cancer: ready for clinical translation! App/ Clin Genet 2013;6:43-52.

27. Schutte M, Seal S, Barfoot R, et al.; Breast Cancer Linkage Consortium. Variants in CHEK2 other than 1100delC do not make a major contribution to breast cancer susceptibility. Am J Hum Genet 2003;72:1023-1028.

28. Weischer M, Bojesen SE, Ellervik C, Tybjaerg-Hansen A, Nordestgaard BG. CHEK2*1100delC genotyping for clinical assessment of breast cancer risk: meta-analyses of 26,000 patient cases and 27,000 controls. J Clin Oncol 2008;26:542-548.

29. Thompson D, Duedal S, Kirner J, et al. Cancer risks and mortality in heterozygous ATM mutation carriers. J Nat/ Cancer Inst 2005;97:813-822.

30. Cybulski C, Górski B, Huzarski T, et al. CHEK2 is a multiorgan cancer susceptibility gene. Am J Hum Genet 2004;75:1131-1135.

31. Helgason H, Rafnar T, Olafsdottir HS, et al. Loss-of-function variants in ATM confer risk of gastric cancer. Nat Genet 2015;47:906-910.

32. Weischer M, Nordestgaard BG, Pharoah P, et al. CHEK2*1100delC heterozygosity in women with breast cancer associated with early death, breast cancer-specific death, and increased risk of a second breast cancer. J Clin Oncol 2012;30:4308-4316

33. Cybulski C, Kluźniak W, Huzarski T, et al.; Polish Hereditary Breast Cancer Consortium. Clinical outcomes in women with breast cancer and a PALB2 mutation: a prospective cohort analysis. Lancet Oncol 2015;16:638-644.

34. Excellence NIfHaC. Familial breast cancer: classification and care of people at risk of familial breast cancer and management of breast cancer and related risks in people with a family history of breast cancer (CG164). http://www.nice.org. uk/CG164. 2013; Accessed 22 March, 2016.

35. Slade I, Riddell D, Turnbull C, Hanson H, Rahman N; MCG programme. Development of cancer genetic services in the UK: a national consultation. Genome Med 2015;7:18.

36. Fischer C, Kuchenbäcker K, Engel C, et al.; German Consortium for Hereditary Breast and Ovarian Cancer. Evaluating the performance of the breast cancer genetic risk models BOADICEA, IBIS, BRCAPRO and Claus for predicting BRCA1/2 mutation carrier probabilities: a study based on 7352 families from the German Hereditary Breast and Ovarian Cancer Consortium. J Med Genet 2013;50:360-367.

37. Goldgar DE, Healey S, Dowty JG, et al.; BCFR; KConFab. Rare variants in the ATM gene and risk of breast cancer. Breast Cancer Res 2011;13:R73.

38. Tavtigian SV, Oefner PJ, Babikyan D, et al.; Australian Cancer Study; Breast Cancer Family Registries (BCFR); Kathleen Cuningham Foundation Consortium for Research into Familial Aspects of Breast Cancer (kConFab). Rare, evolutionarily unlikely missense substitutions in ATM confer increased risk of breast cancer. Am J Hum Genet 2009;85:427-446. 


\section{ORIGINAL RESEARCH ARTICLE}

39. Kilpivaara O, Vahteristo P, Falck J, et al. CHEK2 variant I157T may be associated with increased breast cancer risk. Int J Cancer 2004;111:543-547.

40. Mavaddat N, Pharoah PD, Michailidou K, et al. Prediction of breast cancer risk based on profiling with common genetic variants. J Nat/ Cancer Inst 2015; 107:djv036

41. Domchek SM, Gaudet MM, Stopfer JE, et al. Breast cancer risks in individuals testing negative for a known family mutation in BRCA1 or BRCA2. Breast Cancer Res Treat 2010;119:409-414.

42. Smith A, Moran A, Boyd MC, et al. Phenocopies in BRCA1 and BRCA2 families: evidence for modifier genes and implications for screening. J Med Genet 2007:44:10-15
43. Rowan $E$, Poll A, Narod SA. A prospective study of breast cancer risk in relatives of BRCA1/BRCA2 mutation carriers. J Med Genet 2007;44:e89 (author reply e88).

44. Korde LA, Mueller CM, Loud JT, et al. No evidence of excess breast cancer risk among mutation-negative women from BRCA mutation-positive families. Breast Cancer Res Treat 2011;125:169-173.

45. Kurian AW, Gong GD, John EM, et al. Breast cancer risk for noncarriers of family-specific BRCA1 and BRCA2 mutations: findings from the Breast Cancer Family Registry. J Clin Oncol 2011;29:4505-4509.

46. Harvey SL, Milne RL, McLachlan SA, et al.; kConFab Investigators. Prospective study of breast cancer risk for mutation negative women from BRCA1 or BRCA2 mutation positive families. Breast Cancer Res Treat 2011;130:1057-1061. 